\title{
Hong-Ou-Mandel interferometer with cavities: theory
}

\author{
C. Olindo, M. A. Sagioro, C. H. Monken, and S. Pádua \\ Departamento de Física, Universidade Federal de Minas Gerais, \\ Caixa Postal 702, 30123-970 Belo Horizonte MG, Brazil. \\ A. Delgado \\ Departmento de Física \\ Centro de Optica Cuántica e Información \\ Universidad de Concepción, Chile
}

(Dated: October 31, 2018)

\begin{abstract}
We study the number of coincidences in a Hong-Ou-Mandel interferometer exit whose arms have been supplemented with the addition of one or two optical cavities. The fourth-order correlation function at the beam-splitter exit is calculated. In the regime where the cavity length are larger than the one-photon coherence length, photon coalescence and anti-coalescence interference is observed. Feynman's path diagrams for the indistinguishable processes that lead to quantum interference are presented. As application for the Hong-Ou-Mandel interferometer with two cavities, it is discussed the construction of an optical XOR gate.

PACS numbers:
\end{abstract}

\section{INTRODUCTION}

Spontaneous parametric down-conversion (SPDC) is an important resource for quantum optics studies. In the process of SPDC, a pump $(p)$ laser beam incident upon a nonlinear crystal creates a pair of entangled photons, usually called signal $(s)$ and idler $(i)$ 1]. SPDC was first proposed theoretically by Klyshko in 1969 [2] and demonstrated experimentally by Burnham and Weinberg in 1970 3]. Idler and signal quantum interference in a beam-splitter was first demonstrated by Hong, Ou and Mandel (HOM) in 1987 [4]. In their experiment, signal and idler photons with the same frequency and polarization are combined in a 50/50 beam-splitter (BS) and the output photons are detected at the exit of the beam-splitter by coincidence detection. When the idler and the signal paths are made equal from the crystal to the BS, no coincidence counts are detected at the BS output. This null result is due to a destructive interference between the two indistinguishable paths that the pair can follow to produce coincidences: both photons are reflected at the BS or both photons are transmitted. Since there is a $\pi / 2$ phase shift for the photon reflection probability amplitude at the BS, the probability amplitudes for these two indistinguishable events cancel out and zero coincidence or a minimum, is measured [5]. Therefore, at this point the photon-pair goes to either exit (photon coalescence [6]).

Optical cavities have been used in SPDC quantum optics experiments recently aiming the demonstration of the control of photon coalescence and anti-coalescence via a cavity and the production of time entangled photon-pairs. Sagioro, Olindo, Monken and Pádua 7], have demonstrated experimentally the interference of photons with the same polarization generated by spontaneous parametric down-conversion in a Hong-Ou-Mandel interferometer, after one of them passed through a symmetric cavity, i.e. a cavity whose mirrors have equal reflectivity. In the regime where the cavity length is larger than the one-photon coherence length, photon coalescence and anti-coalescence interference is observed. It is shown that by changing the cavity length, coincidence peaks can be transformed in dips and viceversa, even though the coherence length of the photon wavepacket is smaller than the cavity length. With a different goal, Lu, Campbell and $\mathrm{Ou}$ [8], have generated mode-locked two-photon states by using a Fabry-Pèrot cavity for filtering the wide band light produced by SPDC. The comb-like time correlation of the photon pairs is observed with a HOM interferometer at the exit of the Fabry-Perot cavity. Zavatta, Viciani and Bellini [9], have generated comb-like two-photon entangled states by inserting in one of the arms of a HOM interferometer a Fabry-Perot cavity. In this manuscript, we analyze theoretically a modified version of the Hong-Ou-Mandel interferometer. The optical paths along the arms of the interferometer contain two Fabry-Perot cavities, each one in an arm of the interferometer. These cavities allow the partial transmission and reflection of photons on each arm leading to a deviation of the number $N_{c}$ of coincidences from the standard Hong-Ou-Mandel interferometer.

\section{EXPERIMENTAL SETUP}

The experimental apparatus, represented in Fig. (1), consists of an HOM interferometer [4], and two symmetric cavities formed by two planar dielectric mirrors M5 and M6 (M3 and M4). A 5 mm long $\mathrm{LiIO}_{3}$ crystal, oriented for 


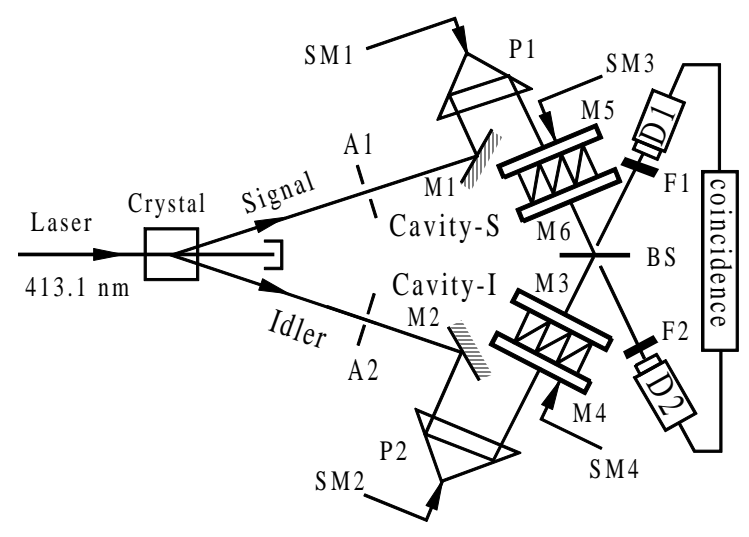

FIG. 1: Experimental scheme of a Hong-Ou-Mandel interferometer in which two Fabry-Pérot cavities are inserted in the arms of the interferometer. The symbols $B S, M, P, S M, A$, and $D$ label beam splitters, mirrors, prisms, step motors, apertures, and detectors, respectively.

type-I phase matching, is pumped by a CW laser oscillating at $413.1 \mathrm{~nm}$. A violet photon from the pump beam is down-converted into two conjugate infrared photons at $826.2 \mathrm{~nm}$ (signal and idler), so that energy and momentum are conserved during this process. Both signal and idler photons are horizontally polarized and propagate at some angle $\theta$ from the pump beam direction. The apertures A1, A2 select out the desired degenerate photon pairs. Both beams are directed by the prisms P1, P2, and mirrors M1, M2 into the two input ports of the 50/50 beam splitter BS. Each beam pass through symmetric Fabry-Perot cavities. Both detectors D1 and D2 are avalanche photodiodes operating in photon counting mode and F1, F2 are equal interference filters centered at $826.2 \mathrm{~nm}$. The prism P1 (P2) is fixed over a translation stage displaceable by a step motor SM1 (SM2) used to change the signal (idler) path length. The step motor SM3 (SM4) is used for moving the mirror M5 (M4) for changing the cavity length $L$.

\section{ANALYTICAL RESULTS}

We consider that the photon-pairs are generated not collinearly (Fig. 11) by SPDC process with the crystal cut for type I phase-matching. In this case, signal and idler photons are generated with the same linear polarization. Suppose, we select the idler and signal photons with the same frequency and equal to half of the frequency of the laser pump beam (degenerate case): $\omega_{i}=\omega_{s}=\omega_{p} / 2$. We also assume that the photon beams are selected by small diameter apertures (see Fig. (1) such that the propagation directions are well selected. In this case, the wavevector dispersion can be neglected and the momentum conservation in the SPDC process considered perfect. We also consider that the pump laser beam is a coherent monochromatic beam, with no dispersion in frequency. The photons are then described by the state [1, 4]

$$
|\Psi\rangle=\int_{0}^{\omega_{p}} d \omega \phi(\omega)|1, \omega\rangle_{i}\left|1, \omega_{p}-\omega\right\rangle_{s}
$$

where the function $\phi(\omega)$ is centered in $\omega_{p} / 2$. In practice $\phi(\omega)$ is defined by interference filters in front the detectors (see Fig. (1). The state $|1, \omega\rangle_{i}\left(\left|1, \omega_{p}-\omega\right\rangle_{s}\right)$ describes one photon of frequency $\omega\left(\omega_{p}-\omega\right)$ in the idler (signal) beam. The contribution from the vacuum cancels out in a photo-coincidence experiment as we have described above, so its contribution have been neglected in Eq. (1). The state $|\Psi\rangle$ can also be cast in the form

$$
\left.|\Psi\rangle=\int_{0}^{\omega_{p}} d \omega \phi(\omega) a_{i}^{\dagger}(\omega) a_{s}^{\dagger}\left(\omega_{p}-\omega\right) \mid \text { vacuum }\right\rangle,
$$

where the operators $a_{i}^{\dagger}(\omega)$ and $a_{s}^{\dagger}\left(\omega_{p}-\omega\right)$ create photons of frequency $\omega$ and $\omega_{p}-\omega$ in the idler and signal modes, respectively.

After being generated, the photons of each beam interact with a Fabry-Perot cavity. The photons can be transmitted or reflected by the cavities. The action of these cavities is similar to a beam splitter [10], that is

$$
\begin{aligned}
& a_{i}^{\dagger}(\omega)=\mu_{i}(\omega) a_{i, t}^{\dagger}(\omega)+\nu_{i}(\omega) a_{i, r}^{\dagger}(\omega), \\
& a_{s}^{\dagger}(\omega)=\mu_{s}(\omega) a_{s, t}^{\dagger}(\omega)+\nu_{s}(\omega) a_{s, r}^{\dagger}(\omega),
\end{aligned}
$$


where the operators $a_{i, t}^{\dagger}(\omega)$ and $a_{i, r}^{\dagger}(\omega)\left(a_{s, t}^{\dagger}(\omega)\right.$ and $\left.a_{s, r}^{\dagger}(\omega)\right)$ describe reflected and transmitted photons of the incoming idler (signal) beam with frequency $\omega$. The coefficients $\mu_{i}(\omega), \nu_{i}(\omega), \mu_{s}(\omega)$, and $\nu_{s}(\omega)$ are the cavities transmission and reflection coefficients for idler and signal beam, respectively. Thereby, the state $|\tilde{\Psi}\rangle$ of the beams after the interaction with the cavities is given by the expression

$$
\begin{aligned}
|\tilde{\Psi}\rangle=\int_{0}^{\omega_{p}} d \omega \phi(\omega) & \left(\mu_{i}(\omega) \mu_{s}\left(\omega_{p}-\omega\right)|1, \omega\rangle_{i, t}\left|1, \omega_{p}-\omega\right\rangle_{s, t}+\mu_{i}(\omega) \nu_{s}\left(\omega_{p}-\omega\right)|1, \omega\rangle_{i, t}\left|1, \omega_{p}-\omega\right\rangle_{s, r}\right. \\
+ & \left.+\nu_{i}(\omega) \mu_{s}\left(\omega_{p}-\omega\right)|1, \omega\rangle_{i, r}\left|1, \omega_{p}-\omega\right\rangle_{s, t}+\nu_{i}(\omega) \nu_{s}\left(\omega_{p}-\omega\right)|1, \omega\rangle_{i, r}\left|1, \omega_{p}-\omega\right\rangle_{s, r}\right)
\end{aligned}
$$

The transmitted beams through the cavities are directed to a 50/50 beam splitter (BS). The two output beams are then detected in coincidence at the BS exit ports. The state of the beams after the cavities, Eq. (5), is a superposition of four mutually orthogonal states. These states describe the reflection or transmission of photons in each beam by the cavities. Consequently, only the transmitted photons of both beams can be detected at the exit of BS.

The field operators at the detectors of the interferometer are 4]

$$
\begin{aligned}
& E_{1}^{+}(t)=\frac{1}{\sqrt{2}}\left[E_{i, t}^{+}\left(t-\tau_{1}-\delta_{i}\right)+i E_{s, t}^{+}\left(t-\tau_{1}-\delta_{s}\right)\right] \\
& E_{2}^{+}(t)=\frac{1}{\sqrt{2}}\left[E_{s, t}^{+}\left(t-\tau_{1}-\delta_{s}\right)+i E_{i, t}^{+}\left(t-\tau_{1}-\delta_{i}\right)\right]
\end{aligned}
$$

where $\tau_{1}$ is the propagation time from mirrors to detectors and $\delta_{i}$ and $\delta_{s}$ are the time delays in the idler and signal beams due to a modification of their optical paths (P1 and P2 can be displaced, see Fig.11).

The field operators at the BS input are given by

$$
\begin{aligned}
& E_{i, t}^{+}(t)=\int d \omega e^{-i \omega t} a_{i, t}(\omega), \\
& E_{s, t}^{+}(t)=\int d \omega e^{-i \omega t} a_{s, t}(\omega) .
\end{aligned}
$$

The probability $P(\tau)$ of detecting one photon on a detector in the time $t$ and a second photon in the other detector, in the time $t+\tau$ is

$$
P(\tau)=\operatorname{Tr}\left(\rho E_{1}^{-}(t) E_{2}^{-}(t+\tau) E_{2}^{+}(t+\tau) E_{1}^{+}(t)\right)
$$

where the density operator $\rho$ describes the state of the electromagnetic field before the beam splitter [1]. The number $N_{c}$ of coincidences can be obtained by integrating this probability in the time interval $\tau$ of the coincidence window.

Due to the purity of the two-photon state, the probability $P(\tau)$ can be calculated as [1]

$$
P(\tau)=\left\langle\Psi_{1}\left|E_{1}^{-}(t) E_{2}^{-}(t+\tau) E_{2}^{+}(t+\tau) E_{1}^{+}(t)\right| \Psi_{1}\right\rangle
$$

where the state

$$
\left|\Psi_{1}\right\rangle=\int_{0}^{\omega_{p}} d \omega \phi(\omega) \mu_{i, \omega} \mu_{s, \omega_{p}-\omega}|1, \omega\rangle_{i, t}\left|1, \omega_{p}-\omega\right\rangle_{s, t}
$$

describes photons transmitted through both cavities.

Let us now consider the state $E_{2}^{+}(t+\tau) E_{1}^{+}(t)\left|\Psi_{1}\right\rangle$. Thereafter the probability $P(\tau)$ can be calculated, according to Eq. (9), as the square of the norm of this state. The action of the operator $E_{2}^{+}(t+\tau) E_{1}^{+}(t)$ onto the state $\left|\Psi_{1}\right\rangle$ is

$$
\begin{aligned}
E_{2}^{+}(t+\tau) E_{1}^{+}(t)\left|\Psi_{1}\right\rangle & =\frac{1}{2}\left(E_{s, t}^{+}\left(t+\tau-\tau_{1}-\delta_{s}\right) E_{i, t}^{+}\left(t-\tau_{1}-\delta_{i}\right)\left|\Psi_{1}\right\rangle\right. \\
& +\frac{i}{2}\left(E_{s, t}^{+}\left(t+\tau-\tau_{1}-\delta_{s}\right) E_{s, t}^{+}\left(t-\tau_{1}-\delta_{s}\right)\left|\Psi_{1}\right\rangle\right. \\
& +\frac{i}{2}\left(E_{i, t}^{+}\left(t+\tau-\tau_{1}-\delta_{i}\right) E_{i, t}^{+}\left(t-\tau_{1}-\delta_{i}\right)\left|\Psi_{1}\right\rangle\right. \\
& -\frac{1}{2}\left(E_{i, t}^{+}\left(t+\tau-\tau_{1}-\delta_{i}\right) E_{s, t}^{+}\left(t-\tau_{1}-\delta_{s}\right)\left|\Psi_{1}\right\rangle .\right.
\end{aligned}
$$

The first term at the r.h.s. of the previous equation can be cast in the form

$$
\frac{1}{2} \iiint d \omega d \omega^{\prime} d \omega^{\prime \prime} e^{-i \omega^{\prime}\left(t+\tau-\tau_{1}-\delta_{s}\right)} e^{-i \omega^{\prime \prime}\left(t-\tau_{1}-\delta_{i}\right)} \phi(\omega) \mu_{i}(\omega) \mu_{s}\left(\omega_{p}-\omega\right) a_{i, t}\left(\omega^{\prime \prime}\right) a_{i, t}^{\dagger}(\omega) a_{s, t}\left(\omega^{\prime}\right) a_{s, t}^{\dagger}\left(\omega_{p}-\omega\right)|\operatorname{vacuum}\rangle
$$


The operators $a_{i, t}\left(\omega^{\prime \prime}\right)$ and $a_{s, t}\left(\omega^{\prime}\right)$ entering in Eq. (12) destroys the vacuum state unless the conditions $\omega^{\prime \prime}=\omega$ and $\omega^{\prime}=\omega_{p}-\omega$ hold. In this case we obtain

$\frac{1}{2}\left(E_{s, t}^{+}\left(t+\tau-\tau_{1}-\delta_{s}\right) E_{i, t}^{+}\left(t-\tau_{1}-\delta_{i}\right)\left|\Psi_{1}\right\rangle=\frac{1}{2} \int d \omega e^{-i\left(\omega_{p}-\omega\right)\left(t+\tau-\tau_{1}-\delta_{s}\right)} e^{-i \omega\left(t-\tau_{1}-\delta_{i}\right)} \phi(\omega) \mu_{i}(\omega) \mu_{s}\left(\omega_{p}-\omega\right)|\operatorname{vacuum}\rangle\right.$.

In a similar way it can be show that

$\frac{1}{2}\left(E_{i, t}^{+}\left(t+\tau-\tau_{1}-\delta_{i}\right) E_{s, t}^{+}\left(t-\tau_{1}-\delta_{s}\right)\left|\Psi_{1}\right\rangle=\frac{1}{2} \int d \omega e^{-i \omega\left(t+\tau-\tau_{1}-\delta_{i}\right)} e^{-i\left(\omega_{p}-\omega\right)\left(t-\tau_{1}-\delta_{s}\right)} \phi(\omega) \mu_{i}(\omega) \mu_{s}\left(\omega_{p}-\omega\right)|\operatorname{vacuum}\rangle\right.$,

being the remaining terms entering in Eq. (11) zero. Thereby, we have

$$
\begin{aligned}
E_{2}^{+}(t+\tau) E_{1}^{+}(t)\left|\Psi_{1}\right\rangle=\frac{1}{2} e^{-i \omega_{p}\left(t-\tau_{1}-\delta_{s}\right)}\left\{e^{-i \omega_{p} \tau} \int_{0}^{\omega_{p}} d \omega e^{i \omega \tau} e^{i \omega\left(\delta_{i}-\delta_{s}\right)} \phi(\omega) \mu_{i}(\omega) \mu_{s}\left(\omega_{p}-\omega\right)\right. \\
\left.-\int_{0}^{\omega_{p}} d \omega e^{-i \omega \tau} e^{i \omega\left(\delta_{i}-\delta_{s}\right)} \phi(\omega) \mu_{i}(\omega) \mu_{s}\left(\omega_{p}-\omega\right)\right\}|\operatorname{vacuum}\rangle .
\end{aligned}
$$

A further simplification of this expression can be obtained by a variable change and a global phase omission. We obtain for Eq. (11)

$$
E_{2}^{+}(t+\tau) E_{1}^{+}(t)\left|\Psi_{1}\right\rangle=\int_{-\frac{\omega_{p}}{2}}^{\frac{\omega_{p}}{2}} d \omega\left(e^{i \tau}-e^{-i \tau}\right) e^{i \omega\left(\delta_{i}-\delta_{s}\right)} \phi\left(\omega+\frac{\omega_{p}}{2}\right) \mu_{i}\left(\omega+\frac{\omega_{p}}{2}\right) \mu_{s}\left(\frac{\omega_{p}}{2}-\omega\right)|\operatorname{vacuum}\rangle .
$$

The transmission coefficients $\mu_{s}$ and $\mu_{i}$ entering in this state are given by

$$
\mu_{j}(\omega)=t_{j}^{2} \sum_{m=0}^{\infty} r_{j}^{2 m} e^{2 i m \omega \tau_{c j}}
$$

where $j=i, s$ denotes the idler or signal beam. $t_{j}$ and $r_{j}$ are the transmission and reflection coefficients of the cavity respectively and $\tau_{c j}=L_{j} / c$ with $L_{j}$ being the $j$ cavity length and $c$ the light velocity. This particular form of the $\mu_{j}$ coefficient arises from considering the cavities as two planar dielectric mirrors with the same reflection and transmission coefficients $\left(r_{j}\right.$ and $\left.t_{j}\right)$ separated by a distance $L_{j}$. Each time a monochromatic plane wave of frequency $\omega$ enters the cavity there are multiple reflections and transmissions of the field on the mirrors. Each time the electromagnetic field of the wave cross a mirror of the cavity, the field is multiplied by $t_{j}$ and each time it is reflected, it is multiplied by $r_{j}$. We must also consider the field propagation between the mirrors (here is the dependence of $\mu_{j}$ on the product of $\omega$ and $L_{j}$ ). Finally, adding all the contributions of the electromagnetic fields that are transmitted by the cavity we get $E_{t}=\mu E_{0}$, where $E_{t}$ is the transmitted field and $E_{0}$ the incident one.

The probability $P(\tau)$ is then given by

$$
\begin{aligned}
P(\tau)= & \frac{K\left(T_{i} T_{s}\right)^{2}}{4} \sum_{m, n, l, q} R_{s}^{m+l} R_{i}^{n+q} e^{i \omega_{p} \tau_{c s}(m-l)} e^{i \omega_{p} \tau_{c i}(n-q)} \int_{-\frac{\omega_{p}}{2}}^{\frac{\omega_{p}}{2}} \int_{-\frac{\omega_{p}}{2}}^{\frac{\omega_{p}}{2}} d \omega d \omega^{\prime} e^{i \omega\left(2 m \tau_{c s}-2 n \tau_{c i}-\delta\right)} e^{-i \omega^{\prime}\left(2 l \tau_{c s}-2 q \tau_{c i}-\delta\right)} \\
& \phi\left(\omega+\frac{\omega_{p}}{2}\right) \phi^{*}\left(\omega^{\prime}+\frac{\omega_{p}}{2}\right)\left(e^{i \tau\left(\omega-\omega^{\prime}\right)}-e^{i \tau\left(\omega+\omega^{\prime}\right)}-e^{-i \tau\left(\omega+\omega^{\prime}\right)}+e^{-i \tau\left(\omega-\omega^{\prime}\right)}\right)
\end{aligned}
$$

where $\delta=\delta_{i}-\delta_{s}$ is the difference of the optical paths of both beams, $T_{j}=\left|t_{j}\right|^{2}$ and $R_{j}=\left|r_{j}\right|^{2}$ are the transmittance and reflectance of the $j$ cavity respectively and $K$ is proportional to the detectors efficiency.

After integrating over $\tau$ and assuming symmetry of $\phi\left(\omega+\frac{\omega_{p}}{2}\right)$ around $\frac{\omega_{p}}{2}$, the number $N_{c}$ of coincidences becomes

$$
\begin{aligned}
N_{c}=\pi K\left(T_{i} T_{s}\right)^{2} & \sum_{m, n, l, q} R_{s}^{m+l} R_{i}^{n+q} \cos \left(\omega_{p} \tau_{c s}(m-l)\right) \cos \left(\omega_{p} \tau_{c i}(n-q)\right) \int_{-\frac{\omega_{p}}{2}}^{\frac{\omega_{p}}{2}} d \omega \\
& {\left[\cos \left(2 \omega\left(\tau_{c s}(m-l)-\tau_{c i}(n-q)\right)\right)-\cos \left(2 \omega\left(\tau_{c s}(m+l)-\tau_{c i}(n+q)\right)-\delta\right)\right]\left|\phi\left(\omega+\frac{\omega_{p}}{2}\right)\right|^{2} .(1) }
\end{aligned}
$$

A further simplification can be achieved considering a gaussian distribution of width $\triangle \omega$ for the function $\phi\left(\omega+\frac{\omega_{p}}{2}\right)$, supposed sufficiently narrow in the interval $\left(-\frac{\omega_{p}}{2}, \frac{\omega_{p}}{2}\right)$ such that the limits of integration can be extended to $(-\infty, \infty)$. Thereby, we finally obtain for the number $N_{c}$ of coincidences the expression

$$
\begin{aligned}
N_{c}=\pi K\left(T_{i} T_{s}\right)^{2} & \sum_{m, n, l, q} R_{s}^{m+l} R_{i}^{n+q} \cos \left(\omega_{p} \tau_{c s}(m-l)\right) \cos \left(\omega_{p} \tau_{c i}(n-q)\right) \\
& {\left[e^{\left(-\triangle \omega^{2}\left(\tau_{c s}(m-l)-\tau_{c i}(n-q)\right)^{2}\right)}-e^{\left(-\triangle \omega^{2}\left(\tau_{c s}(m+l)-\tau_{c i}(n+q)-\delta\right)^{2}\right)}\right] . }
\end{aligned}
$$




\section{HOM WITH ONE CAVITY}

Here we study interferometric properties of HOM interferometer with one cavity. To obtain the number of coincidences $N_{c}$ in this particular case we make $R_{s}=0, T_{s}=1$ and $\tau_{c s}=0$ in Eq. (20). Thereby, we consider a single cavity in the path of the idler beam. The number of coincidences becomes

$$
N_{c}=T^{2} \sum_{n, q} R^{n+q} \cos \left(\omega_{p} \tau_{c}(n-q)\right)\left(e^{-\Delta \omega^{2} \tau_{c}^{2}(n-q)^{2}}-e^{-\Delta \omega^{2}\left(\tau_{c}(n+q)-\delta\right)^{2}}\right),
$$

with $T=T_{i}, R=R_{i}, \tau_{c}=\tau_{c i}$ and the constant $\pi K$ was removed because we are interested in the coincidence rate, not in the number of coincidences itself. In particular, making $T=1, R=0$ and $\tau_{c}=0$ we recover from Eq. (21) the well known expression for the number of coincidences of the HOM interferometer without cavities, that is

$$
N_{c}=1-e^{-\Delta \omega^{2} \delta^{2}} .
$$

Before proceeding with the analysis of Eq. (21) we make a change of variables. This equation becomes

$$
N_{c}=T^{2} \sum_{n, q} R^{n+q} \cos \left(\frac{2 \pi L}{\lambda_{p}}(n-q)\right) e^{-\left(\frac{2 \pi \Delta \lambda L}{\lambda^{2}}(n-q)\right)^{2}}-T^{2} \sum_{n, q} R^{n+q} \cos \left(\frac{2 \pi L}{\lambda_{p}}(n-q)\right) e^{-\left(\frac{2 \pi c \Delta \lambda}{\lambda^{2}}\right)^{2}\left(\frac{L}{c}(n+q)-\delta\right)^{2}}
$$

where $\lambda$ is the central wavelength of the twin photons wavepackets, $\lambda_{p}$ is the pump wavelength, $\triangle \lambda$ characterizes the filter in front of the detectors, $c$ is the speed of light and $L$ is the length of the cavity.

Figures (2), (31) and (43) show the behavior of the number of coincidences, according to Eq. (23), as a function of the delay $\delta$. The values for the different parameters entering in this equation were taken from the experimental data reported by Sagioro et al 7]. In Fig. (2) the length $L$ of the cavity was chosen as an integer multiple of half of the central wavelength of the photon wavepacket. In this case, the curve is composed of a mesa function intersected by equally separated valleys. In Fig. (3) the length of the cavity was chosen as half integer multiple of half of the central wavelength of the photon wavepacket. In this case the curve is composed of a mesa function intersected by alternated valleys and peaks. We refer to these cases as resonant and anti-resonant respectively. A different situation is depicted in Fig. (4a) which shows the number of coincidences when the conditions for resonance or anti-resonance are not fulfilled. In this particular case peaks and valleys do not alternate following a regular pattern. Fig. (4) also shows the behavior of the number of coincidences when we change the value of the other parameters entering in Eq. (23). In Fig. (4) the value of $R$ has been increased from 0.7 to 0.9. The only noticeable effect is a change in the value of the mesa function and on the amplitudes of valleys and peaks. The positions of peaks and valleys remains unchanged. In Fig. (44) we change $\Delta \lambda$ from $8 \mathrm{~nm}$ to $4 \mathrm{~nm}$ leaving the other parameters as in Fig. (45). In this case increases the width of valleys and peaks leaving the other features of the number of coincidences unchanged. Figure (4/) shows the modification of the number of coincidences when the wavelength is changed from $826.2 \mathrm{~nm}$ to $1200 \mathrm{~nm}$. Notice that some of the valleys and peaks appears to be absent. In Fig. (46) the parameters are as in Fig. (45) with $L=0.44444$. Finally, in Fig. (4F) $L$ and $\lambda$ were changed while keeping the ratio $L / \lambda$ fix. In this case the number of coincidences behaves like in Fig. (4)).

The value of the mesa function can be obtained easily. We consider $N_{c}$ as a function of the delay $\delta$. Thus, the first term entering in Eq. (23) turns out to be a constant. Since, for the values of the parameters that we consider, the ratio $2 \pi \Delta \lambda L / \lambda^{2}$ is larger than 30 the exponential function in the first term of Eq. (23) approximately vanishes for values of $n-q \neq 0$. Thereby, Eq. (23) becomes

$$
N_{c}=\frac{T^{2}}{1-R^{2}}-T^{2} \sum_{n, q} R^{n+q} \cos \left(\frac{2 \pi L}{\lambda_{p}}(n-q)\right) e^{-\left(\frac{2 \pi c \Delta \lambda}{\lambda^{2}}\right)^{2}\left(\frac{L}{c}(n+q)-\delta\right)^{2}} .
$$

Figure (6) shows the number of coincidences as a function of the reflectance $R$. The curve coincides exactly with the function $T^{2} /\left(1-R^{2}\right)$. Let us see where this function comes from. The probability of one photon to be transmitted through a cavity is $T$ and to be reflected is $R$. Summing over all the possibilities (a photon crossing the cavity directly, a second reflecting inside twice before crossing it, a third reflecting four times and so on), and obtain the total probability for crossing the photons $(P): P=T^{2}+T^{2} R^{2}+T^{2} R^{4}+T^{2} R^{6}+\ldots=\frac{T^{2}}{1-R^{2}}$. This result shows that, when the longitudinal coherence length $\lambda^{2} / \triangle \lambda$ of the single photons is much smaller than the cavity length $L$, the photon acts like particles with probability $T$ for crossing an obstacle and probability $R$ to be reflected, i.e. there is no interference between a photon with itself, as there was in a traditional Fabry-Perot cavity.

The second term in Eq. (23) composes of a sum over unnormalized gaussian functions $e^{-(\delta-\tilde{\delta})^{2} / 2 \sigma^{2}}$ centered at $\tilde{\delta}_{n+q}=(L / c)(n+q)$ with standard deviation $\sigma=\lambda^{2} /(2 \sqrt{2} \pi c \Delta \lambda)$, which is independent of $n$ and $q$. Let us note 

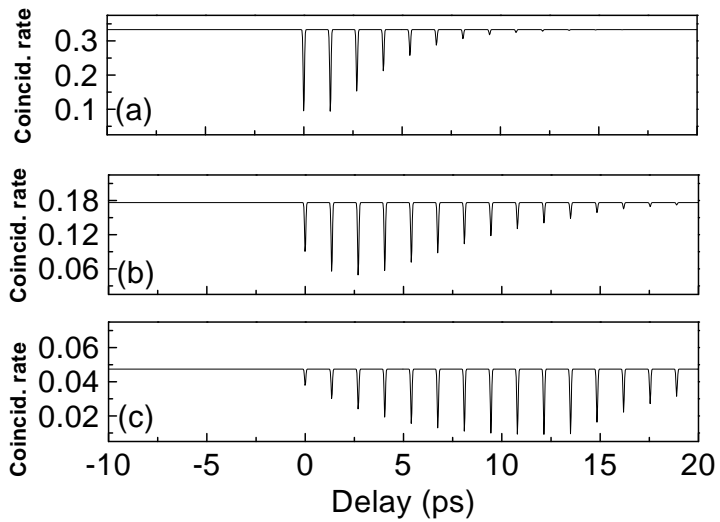

FIG. 2: In all three graphics the wavelength of the degenerate photons $(\lambda)$ was $826.2 \mathrm{~nm}$ and the interference filters in front of the detectors had bandwidth $(\Delta \lambda)$ equal to $8 \mathrm{~nm}$. In all three cases $L$ was equal to $0.404838 \mathrm{~mm}$ (to achieve the resonant situation). In (a) $R=0.5$, in (b) $R=0.7$, in (c) $R=0.9$ and $R+T=1$ (no losses).

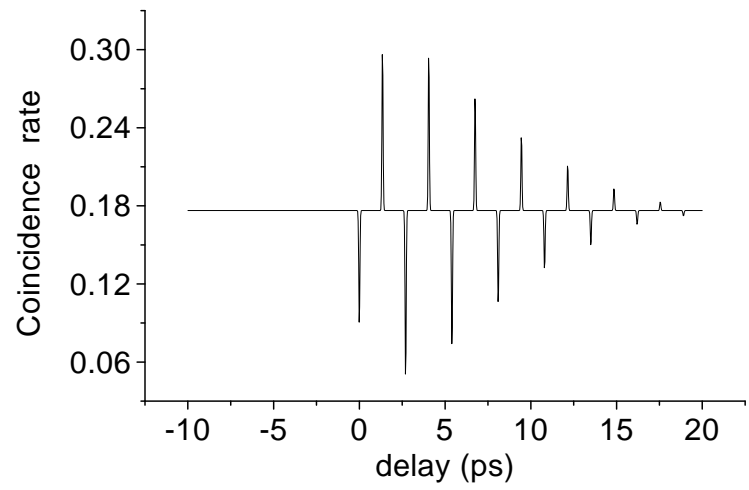

FIG. 3: The setup parameters are: $\lambda=826.2 \mathrm{~nm}, \Delta \lambda=8 \mathrm{~nm}, R=0.7$ and $L=0.4050447 \mathrm{~mm}$.

that the distance between the centers of two consecutive gaussian functions is given by $L / c$. The overlap between two consecutive gaussian functions is given by

$$
\int_{-\infty}^{+\infty} d \delta e^{-\left(\frac{\delta-\tilde{\delta}_{n+q}}{\sqrt{2} \sigma}\right)^{2}} e^{-\left(\frac{\delta-\tilde{\delta}_{n+q+1}}{\sqrt{2} \sigma}\right)^{2}}=\sqrt{\pi} \sigma e^{-\left(\frac{\tilde{\delta}_{n+q}-\tilde{\delta}_{n+q+1}}{2 \sigma}\right)^{2}}
$$

being its value tenth of picoseconds for the value of the parameters here considered. Thus, it is possible to distinguish consecutive gaussian functions. These gaussian functions lead to the peaks and valleys in figures (2), (3) and (4). Peaks or valleys appear for values of $\delta$ fulfilling the condition

$$
\delta=\frac{L}{c}(n+q)
$$

with a maximum amplitude given by

$$
N_{c}(j)=\frac{T^{2}}{1-R^{2}}-T^{2} \sum_{n, q} R^{n+q} \cos \left(\frac{2 \pi L}{\lambda_{p}}(n-q)\right) .
$$

where $j$ is the order of the peak or valley $\left(j=1\right.$ for the first, in $\delta=0 ; j=2$ for the second, in $\delta=\tau_{c} ; j=3$ for the third, in $\delta=2 \tau_{c}$ and so on) and the summation is for all $n+q=j-1$.

Let us now consider for instance the condition for the resonant case. In this case, the cosine function entering in Eq. (27) is always one. Thereby, the amplitude of the valleys becomes

$$
N_{c}(j)=\frac{T^{2}}{1-R^{2}}-T^{2} R^{j-1} j .
$$



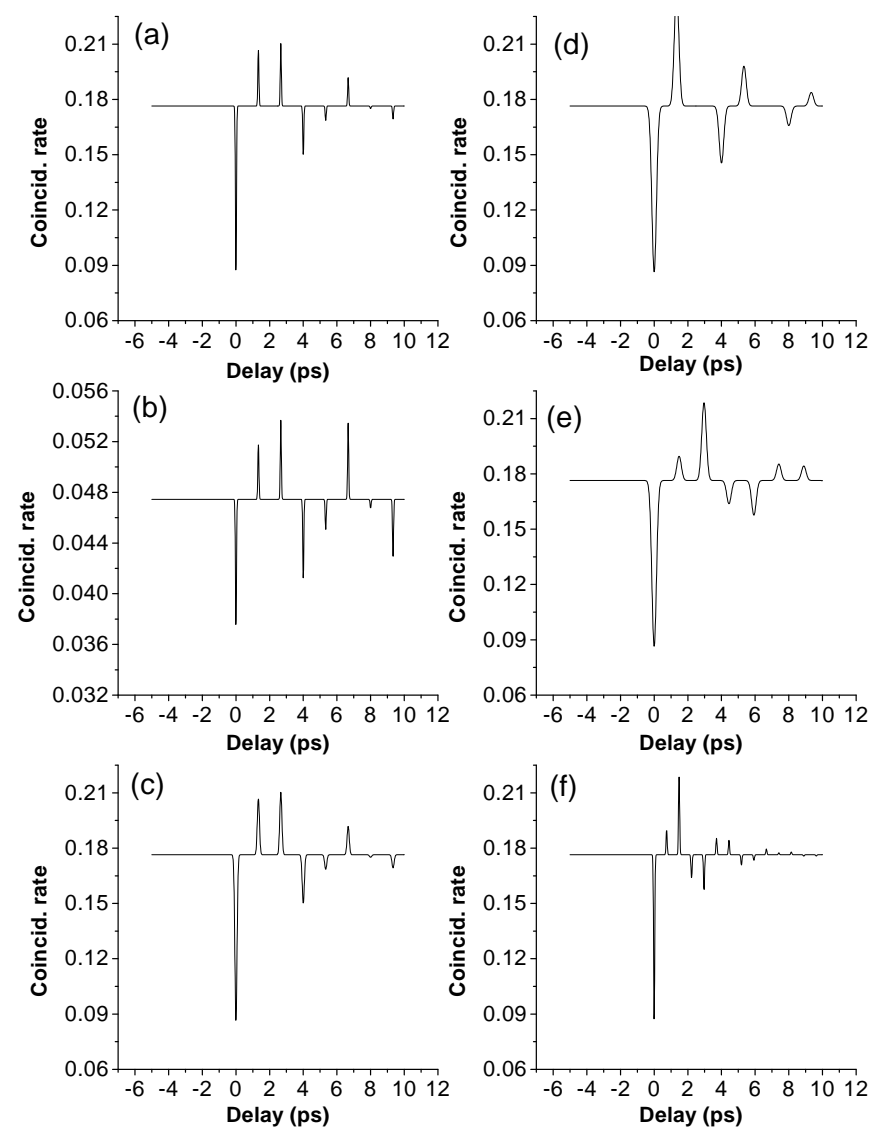

FIG. 4: We begin in (a) with $\lambda=826.2 \mathrm{~nm}, \Delta \lambda=8 \mathrm{~nm}, L=0.4 \mathrm{~mm}, R=0.7$. In (b) we change only $R$ to 0.9 . In (c) we come back $R$ to 0.7 , maintaining all the other parameters the same, and change $\Delta \lambda$ to $4 \mathrm{~nm}$. In (d), in relation to (c), we only change $\lambda$ to $1200 \mathrm{~nm}$. In (e), we made $L$ equal to $0.44444 \mathrm{~mm}$. Finally in (f), we change $\lambda$ to $600 \mathrm{~nm}$ and $L$ to $0.22222 \mathrm{~mm}$.

In the anti-resonant case the amplitude of the peaks and valleys is given by

$$
N_{c}(j)=\frac{T^{2}}{1-R^{2}}-T^{2}(-R)^{j-1} j .
$$

The origin of peaks and valleys can be illustrated by considering the diagrams depicted in Fig. (7). Fig. (7) shows the signal beam with a delay $\delta=\tau_{c}$ which is produced by changing the length of the optical path (see Fig. (10). The photons of the idler beam are transmitted through the cavity without being reflected by the mirrors of the cavity. Both beams are reflected at the beam splitter. The overall effect is that detector D2 detects a photon a time $\tau_{c}$ before detector D1. The situation depicted in Fig. (7h') is slightly different but the overall effect remains unchanged. In this case photons of the idler beam reflect twice inside the cavity but photons of both beams are transmitted at the beam splitter, so that detector D2 detects the photons a time $\tau_{c}$ before detector D1. The situation is similar in the cases of Fig. (7b) and (77'), the only difference is that detector D1 detects photons a time $\tau_{c}$ before detector D2. These four cases, which are partially distinguishable due to their different probabilities, contribute to the same peak or valley at $\delta=\tau_{c}$ in the coincidence detection. The interference at $\delta=2 \tau_{c}$ can also be explained in these terms. Figures (17)), (7)'), (7/ $),\left(7 \mathrm{~d}^{\prime}\right),(\mathbf{7})$ and (7)') show all the possible processes contributing in this case. Notice that in the process depicted in Fig. (7) the photons overlap at the beam splitter, but in the other processes there is no such an overlap between the photons. When $\delta=0$, the only possible process is the overlap between the photons at the beam splitter 


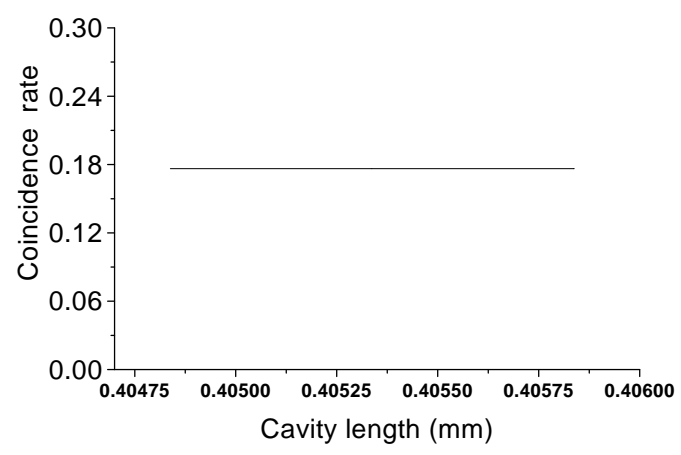

FIG. 5: Graphic showing coincidence rate versus cavity length. We begin with $L=0.404838 \mathrm{~mm}$ and fix the delay in 0.66733 ps, which is approximately in the middle between the first and the second interferences (platform), and vary $L$ just a few lambdas. The parameters are $R=0.7, \lambda=826.2 \mathrm{~nm}$ and $\Delta \lambda=8 \mathrm{~nm}$.

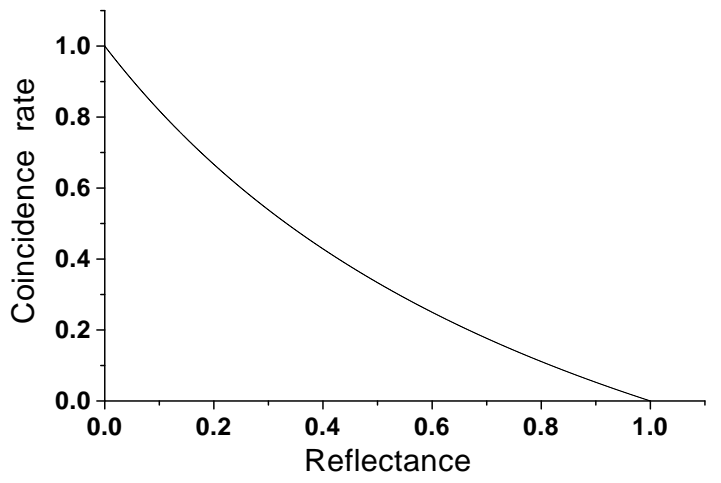

FIG. 6: Graphic showing the coincidence rate versus reflectance $(R)$. We put $L=0.4 \mathrm{~mm}$, the delay in $0.66733 \mathrm{ps}$, which is approximately in the middle between the first and the second interferences (platform); $\lambda=826.2 \mathrm{~nm}$ and $\Delta \lambda=8 \mathrm{~nm}$.

caused by the direct crossing of photons of the idler beam through the cavity.

Figure 7 shows why the interferences always happen when $\delta$ is a multiple of $\tau_{c}$. By analyzing Eq. (21) we deduce the coincidence rate value for each interference region:

$$
N_{c}(j)=\frac{T^{2}}{1-R^{2}}-T^{2} \sum_{n, q} R^{n+q} \cos \left[\omega_{p} \tau_{c}(n-q)\right] .
$$

Where $j$ is the order of the interference region $\left(j=1\right.$ for the first, in $\delta=0 ; j=2$ for the second, in $\delta=\tau_{c} ; j=3$ for the third, in $\delta=2 \tau_{c}$ and so on) and the summation is for all $n+q=j-1$.

Now we would like to come back to Fig. 2] remember that increasing $R$ the deepest valley is each time more distant from the origin, we will give a brief explication for such behavior. Look at Fig. 7 parts (a) and (b) are associated with the second interference region $\left(\delta=\tau_{c}\right)$, parts (c), (d) and (e) are associated with the third one $\left(\delta=2 \tau_{c}\right)$. We could write out parts (f), (g), (h) and (i) associated with the fourth interference region, if we want to, and so on. Notice that increasing the order of the interference region, the number of pairs of interfering processes increases the same quantity. On the other hand, the probability of occurrence of these processes decreases. The case shown in Fig. 2 is the resonant one, when all the interference contributions are destructive. To see this, take Eq. (21) and make $\omega_{p} \tau_{c}=E \pi$, where $\mathrm{E}$ is an even number (resonance condition). By analyzing the same Eq. (21), we conclude that the probability of occurring determinate pair of interference process is proportional to $R^{(j-1)}$, where $R$ is the cavity mirror reflectance and $j$ is the order of the interference region. The number of pairs of interference processes in each interference region is $j$, so the total probability of occurring determinate interference region is proportional to $j R^{(j-1)}, P(j, R) \propto j R^{(j-1)}$. The highest $P(j, R)$ corresponds to the deepest valley (in the resonant case). Once we have $P(j, R)$ it is easy to show that its highest value, when you increase $R$ is for a higher $j$. This highest value for $P(j, R)$ when $R \rightarrow 1$ is when $j \rightarrow \infty$. 


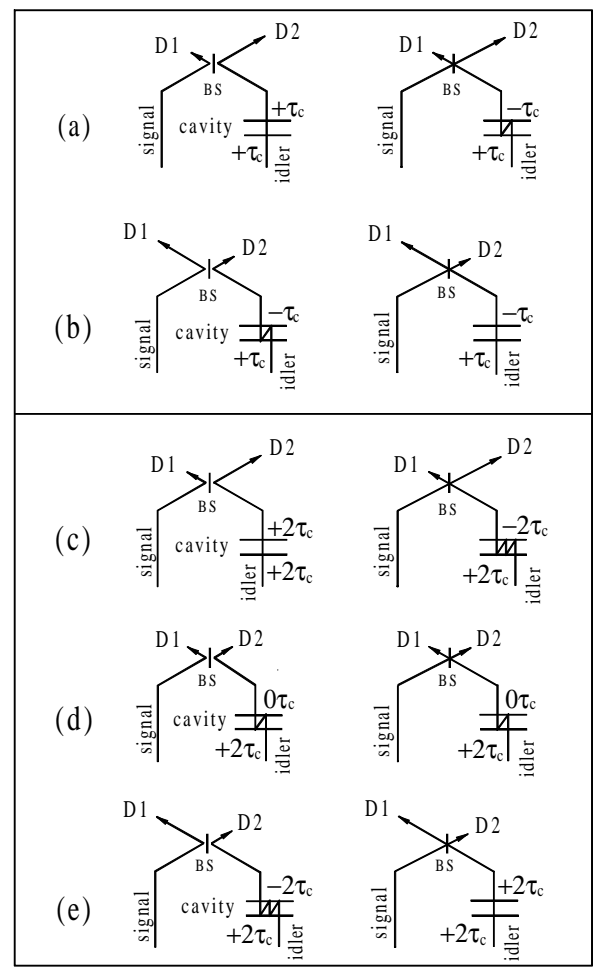

FIG. 7: (a) and (b) are Feynman's path diagrams associated with the second interference region $\left(\delta=\tau_{c}\right)$. (c), (d) and (e) are Feynman's path diagrams associated with the third interference region $\left(\delta=2 \tau_{c}\right)$.

\section{HOM WITH TWO CAVITIES}

Now we will show some simulations using the complete Eq. 20] with the constant $\pi K$ removed. Figure 8 shows the interference patterns when two cavities are present in the interferometer.

Notice that in Fig. $8 \mathrm{a}$ and in Fig. $8 \mathrm{c}$ we have symmetric patterns with respect to the origin, but we do not have this symmetry in Fig. 8 b. Notice also that there are interferences for negative delays, not only a platform as in the one cavity problem (Fig. 24). Finally notice that in Fig. 8 a and Fig. 8 c, when $\delta=0$ we get $100 \%$ of coalescence. This totally destructive interference is not possible, when the longitudinal coherence of the photons is much smaller than the cavity length, using only one cavity.

Let us see what happens when the cavities are unbalanced (Fig. 9).

In Fig. 9 the coincidence pattern is composed only of valleys (both cavities are resonant), but the platform changes its value from what it was in Fig. [ 8 a because the percentages of crossing photons are different $\left(R_{i}=0.7\right.$ and $\left.R_{s}=0.4\right)$.

Now we take the case of identical cavities without resonances and anti-resonances (Fig. 10).

See in Fig. [10] that the coincidence pattern is symmetric with respect to the origin, because $L_{i}=L_{s}$, but at this time the coincidence rate platform has a lower value than in Fig. 8 a and Fig. [ 8 c, because the cavities are neither resonant nor anti-resonant.

In the one cavity case, the platform does not depend on $L$ and has an special meaning (Fig. 55). How would this be with two cavities? See Fig. [1].

In Fig. 11] a we begin with $L_{i}=0.404838 \mathrm{~mm}$ (resonant) and vary it just a few lambdas, passing by anti-resonances, intermediate cases, coming back to resonances and so on. Notice now that, unlike Fig. [5] the platform depends on $L$. Its value is maximum exactly when $L_{i}$ is resonant (because $L_{s}$ is resonant too) and is minimum when $L_{i}$ is anti-resonant. With two cavities the platform does not mean the percentage of crossing photons anymore, i.e. you never are free of interferences, any place you put the delay, including the platform, you have some kind of interference (this will be better understood when we study the paths interference Feynman's diagrams for the two cavities). The distance between consecutive maximums is $\lambda / 2$. In Fig. 11] b, the only difference from Fig. 11] is that we fixed $L_{s}$ in $L_{s}=0.4 \mathrm{~mm}$ (neither resonant nor anti-resonant) and began with $L_{i}=0.4 \mathrm{~mm}$. The procedure was the same (to vary $L_{i}$ just a few lambdas). Now the distance between consecutive minimums is $\lambda / 4$. 
(a)
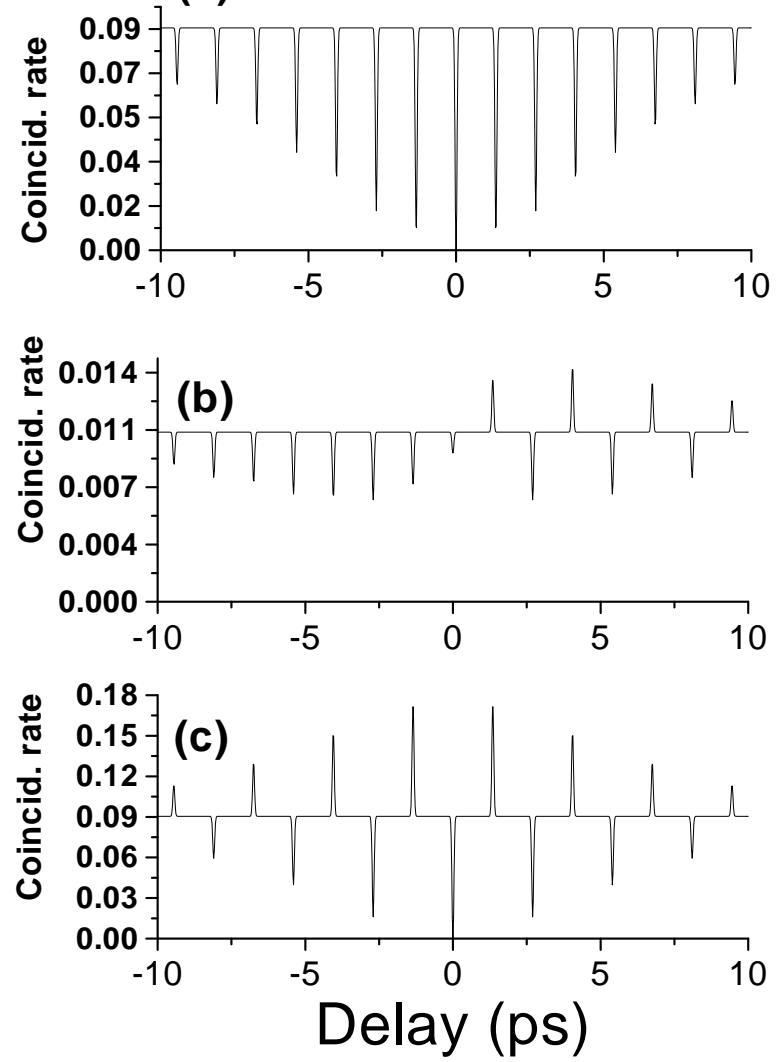

FIG. 8: In this graphic both cavities have the parameters: $R_{i}=R_{s}=0.7, \lambda_{i}=\lambda_{s}=826.2 \mathrm{~nm}$ and $\Delta \lambda=8 \mathrm{~nm}$. In (a) $L_{i}=L_{s}=0.404838 \mathrm{~mm}$, both cavities are resonant with the twin photons. In (b) $L_{s}=0.404838 \mathrm{~mm}$ (resonant), $L_{i}=0.4050447 \mathrm{~mm}$ (anti-resonant). In (c) $L_{s}=L_{i}=0.4050447 \mathrm{~mm}$, both cavities are anti-resonant.

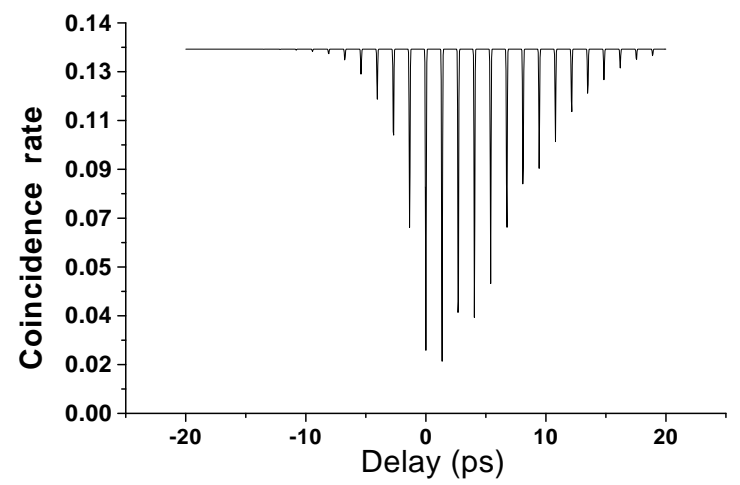

FIG. 9: The parameters are $\lambda=826.2 \mathrm{~nm}, \Delta \lambda=8 \mathrm{~nm}, L_{i}=L_{s}=0.404838 \mathrm{~mm}$ (both resonant), $R_{i}=0.7$ and $R_{s}=0.4$.

We can explain the platform variation with $L$ by analyzing Fig. 12

The reasons for the presence of peaks and dips in the interference pattern of the HOM interferometer with two cavities are the same presented in Fig. 7 you do not know what photon arrives in a detector first. But the coincidence rate platform with just one cavity does not depend on $L$ (Fig. 5). When you have two cavities, the platform itself depends on $L$ (Fig. 11). We could explain this dependence in a general fashion, but, to make the explication easier to understand, we took a particular case. In Fig. 12 $\tau_{c s} \simeq \tau_{c i}$ or $\tau_{c s}=\tau_{c i}$, so we call them $\tau_{c}$. We began with photon idler advanced by $0.5 \tau_{c}$ with respect to photon signal (we made use of prisms P1 and P2 to do so, see Fig. 1). 


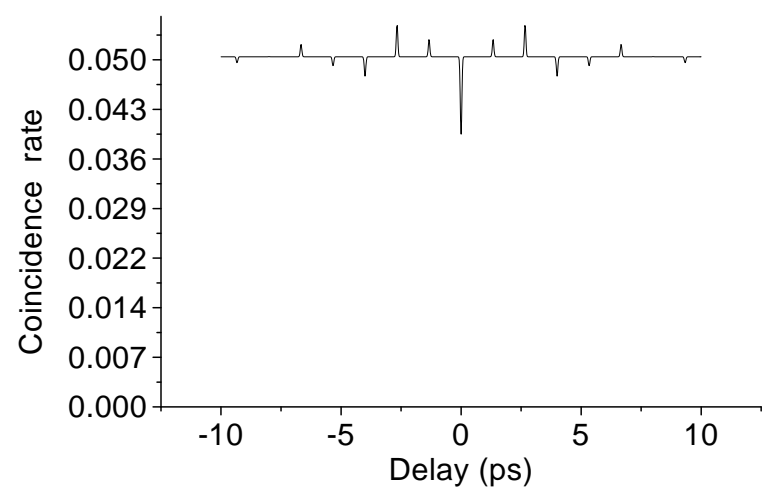

FIG. 10: The parameters are $\lambda=826.2 \mathrm{~nm}, \Delta \lambda=8 \mathrm{~nm}, R_{i}=R_{s}=0.7$ and $L_{i}=L_{s}=0.4$ mm (neither resonant nor anti-resonant).
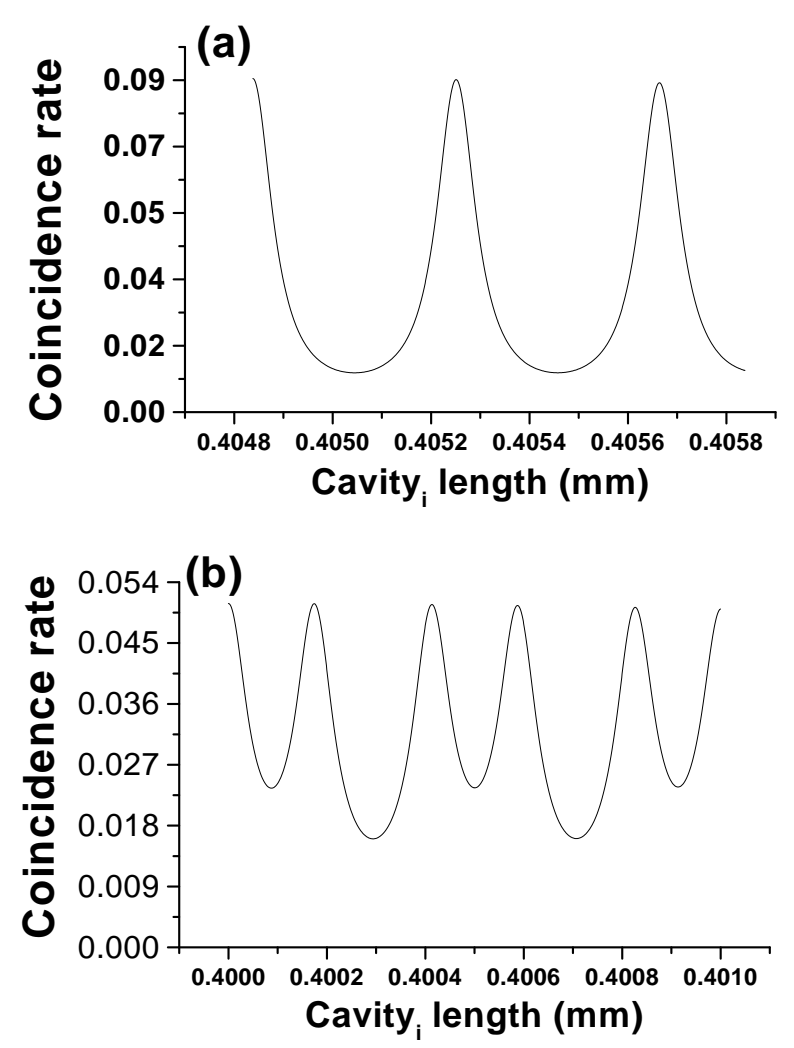

FIG. 11: Graphics showing coincidence rate versus $L_{i}$. All the other parameters are kept fix: $R_{i}=R_{s}=0.7, \lambda=826.2 \mathrm{~nm}$, $\Delta \lambda=8 \mathrm{~nm}$, delay $=0.66733 \mathrm{ps}$ (platform), in (a) $L_{s}=0.404838 \mathrm{~mm}$ (resonant), in (b) $L_{s}=0.4 \mathrm{~mm}$ (neither resonant nor anti-resonant).

In Fig. 12] a, both photons can cross its cavities directly or both can reflect inside the cavities twice before crossing them or (this is not shown here) they can reflect inside their cavities four times before crossing the cavities and so on; in all these cases photon idler still would be $0.5 \tau_{c}$ advanced. In Fig. $12 \mathrm{~b}$, because of the different amounts of reflections between the photons inside their cavities, photon signal arrives 1.5 $\tau_{c}$ before photon idler in BS, and this can happen by various different ways. If the difference between the clicks at the detectors is $0.5 \tau_{c}, 2.5 \tau_{c}, 4.5 \tau_{c}$ and so on, you know that was photon idler that arrived first, if the difference is $1.5 \tau_{c}, 3.5 \tau_{c}, 5.5 \tau_{c}$ and so on, you know that 


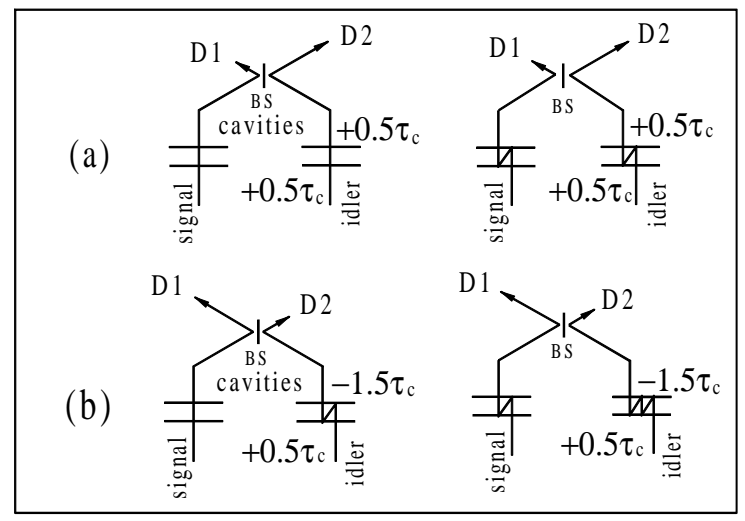

FIG. 12: Paths interference Feynman's diagrams for two cavities, explaining the platform variation with $L$. Photon idler begin $0.5 \tau_{c}$ advanced in relation to photon signal. In (a) it remains $0.5 \tau_{c}$ advanced, after both twin photons cross their cavities. In (b), photon signal, after both photons cross their cavities, is advanced $1.5 \tau_{c}$ in relation to photon idler.

was photon signal that arrived first. Unlike the interferences that causes the peaks and dips, when you do not know with certainty what photon arrives first at a detector, in this kind of interference (that makes the platform vary) you know what photon arrives first, but you do not know which process has happened before the arriving. Resuming: in Fig. 12 all process in line (a) interfere, all process in line (b) interfere and so on, this is the very reason for the fluctuation of the platform.

\section{APPLICATIONS: CONTROL NOT GATE}

This setup (Fig. 1) is suitable for applications in information problems. For example: we can construct an optical $X O R$ gate [11], in a similar way as shown in ref. [12]. In their case the control was made by changing electro-optically the birefringence of two quartz plates with different lengths inserted at each arm of the HOM. In our case the control is made by changing the length of the cavities. The HOM interference pattern changes if the length of each cavity is an integer multiple of half wavelength ("resonance") or a semi-integer multiple of half wavelength ("anti-resonance"). The logical bits are encoded in the resonances/antiresonances of the cavities with the photon central wavelength similarly to the phases of photon 1 and 2 in the setup of Becker et al. [12]. Calculations concerning two cavities, each one in one of the arms of the interferometer, showed above the behavior of the coincidence counts. Consider that the cavities are nearly equal in length. If both central wavelength of idler and signal photons are resonant or anti-resonant with their cavities, you have a symmetric interference pattern, in relation to $\delta=0$, in the coincidence counts. But if one of the photons is resonant with its cavity and the other is anti-resonant with its one, it is obtained a not symmetric coincidence pattern (in relation to $\delta=0$ ), see Fig. 8 Now let us identify input bit 0 with the resonance and input bit 1 with the anti-resonance. We also identify output bit 0 with the symmetric pattern in the coincidences and output bit 1 with the not symmetric pattern. With these identifications, it is easy to simulate a logical XOR operation, see table 【

\begin{tabular}{l}
\hline \multicolumn{1}{c|}{ Table for XOR gate } \\
\begin{tabular}{|rr|rr||ll|}
\hline \multicolumn{2}{|c|}{ Idler } & \multicolumn{2}{c|}{$\begin{array}{c}\text { Signal } \\
\text { bit cavity }\end{array}$} & \multicolumn{2}{c|}{ Results } \\
\hline 0 & res & 0 & res & SY & 0 \\
0 & res & 1 & ant & NS & 1 \\
1 & ant & 0 & res & NS & 1 \\
1 & ant & 1 & ant & SY & 0 \\
\hline
\end{tabular}
\end{tabular}

TABLE I: Table showing the behavior of a logical XOR operation. "res" stands for resonant, "ant" for anti-resonant, "SY" stands for the symmetric coincidence pattern and "NS" for the not symmetric one.

Table \ shows that we can choose photon idler as the control and photon signal as the target, for simulating the 
XOR gate, or vice-versa. This interchanging between control and target is very useful in constructing logical circuits.

\section{CONCLUSION}

We started making the calculations for the coincidence measures in the Hong-Ou-Mandel interferometer with two symmetric cavities, each one put in one arm of the interferometer. Using the resulting equation we made some simulations. It was easy to reduce the general equation for two cavities to the more specific one for just one cavity. One interesting result in this case (one cavity) is the observation of coalescence and anti-coalescence, both comportment are determinate by the rate between the cavity length and the wavelength of the photon that cross it, although the photon coherence length is smaller than the cavity length. But, the main conclusion concerning anti-coalescence is that this is only possible due to the non-overlapping processes happening from the second interference region (see Fig. 7 $\mathrm{a}, \mathrm{b}$ ) in ahead. If the photons overlap in the beam splitter (having the same polarization, frequency and transverse mode) they necessarily leave the beam splitter by the same output, giving origin to no coincidence. In the two cavities case, there are two results that deserve to be pointing. The first is the null result for the coincidences when $\delta=0$ (Fig. [8 a,c), this was not possible in the one cavity case. The second is the variation of the platform with the changing in the cavities lengths, this is due a new kind of interference that either did not happen in the one cavity case. Finally we show that is possible to construct a XOR gate using our apparatus. In fact, if you prefer, instead of using as outgoing bits, SY standing for bit 0 and NS for bit 1 (see table 1 last collum) you can use the value of the platform (see Fig. 8), all you have to do is to stop the delay in the platform, e.g. $\delta=0.66733$ ps in that case, calibrate your system, and you are ready to use the platform value as outgoing bits, for example: the highest value can stand for bit 0 and the lowest one for bit 1 .

\section{ACKNOWLEDGEMENTS}

This work was supported by CNPq, Pronex-Semicondutores and Milênio-Informação Quântica. We would like to thank S. P. Walborn for pointing us ref. [12]. A.D. was supported by Fundación Andes.

[1] L. Mandel and E. Wolf, Optical Coherence and Quantum Optics, Cambridge University Press, Cambridge (1995).

[2] D. N. Klyshko, JETP 28, 522 (1969).

[3] D. C. Burnham and D. L. Weinberg, Phys. Rev. Lett. 25, 84 (1970).

[4] C. K. Hong, Z. Y. Ou, and L. Mandel, Phys. Rev. Lett. 37, 2044 (1987).

[5] Z. Y. Ou and L. Mandel, Phys. Rev. Lett. 57, 66 (1989); A. M. Steinberg and R. Y. Chiao, Phys. Rev. A 49, 3283 (1994).

[6] Kaige Wang, and Shiyao Zhu, Euro. Phys. Lett. 64, 22 (2003).

[7] M. A. Sagioro, C. Olindo, C. H. Monken, and S. Pádua, Phys. Rev. A 69, 053817 (2004). (1994).

[8] Y. J. Lu, R. L. Campbell, and Z.Y. Ou, Phys. Rev. Lett. 91, 163602 (2003).

[9] A. Zavatta, S. Viciani, and M. Bellini, arXiv:quant-ph/0312082 (2003).

[10] C. H. Monken, and G. A. Barbosa, Opt. Commun. 99, 152 (1993).

[11] M. A. Nielsen, and I. L. Chuang, Quantum Communication and Quantum Information, Cambridge University Press, Cambridge (2000).

[12] H. Becker, K. Schmid, W. Dultz, W. Martienssen, H. Roskos, in Quantum Information Processing, edited by Gerd Leuchs and Thomas Beth (Wiley-VCH, Weinhein, 2003), pag. 301.

[13] Grant R. Fowles, Introduction to Modern Optics, Holt, Rinehart and Winston, Inc., New York (1975) 